\title{
Effect of prostatic apex shape (Lee types) and urethral sphincter length in preoperative MRI on very early continence rates after radical prostatectomy
}

\author{
Mike Wenzel $^{1,2}{ }^{(0} \cdot$ Felix Preisser $^{1} \cdot$ Matthias Mueller $^{1} \cdot$ Lena H. Theissen $^{1} \cdot$ Maria N. Welte $^{1} \cdot$ Benedikt Hoeh $^{1}$. \\ Clara Humke $^{1}$ - Simon Bernatz ${ }^{3}$. Boris Bodelle ${ }^{3}$. Christoph Würnschimmel ${ }^{2,4}$. Derya Tilki ${ }^{4,5} \cdot$ Hartwig Huland $^{4}$. \\ Markus Graefen ${ }^{4}$. Frederik C. Roos ${ }^{1}$. Andreas Becker ${ }^{1}$ Pierre I. Karakiewicz ${ }^{2}$ Felix K. H. Chun ${ }^{1}$. Luis A. Kluth ${ }^{1}$. \\ Philipp Mandel ${ }^{1}$
}

Received: 27 November 2020 / Accepted: 8 February 2021 / Published online: 19 February 2021

(c) The Author(s) 2021, corrected publication 2021

\begin{abstract}
Purpose To test the effect of anatomic variants of the prostatic apex overlapping the membranous urethra (Lee type classification), as well as median urethral sphincter length (USL) in preoperative multiparametric magnetic resonance imaging (mpMRI) on the very early continence in open (ORP) and robotic-assisted radical prostatectomy (RARP) patients.

Methods In 128 consecutive patients (01/2018-12/2019), USL and the prostatic apex classified according to Lee types A-D in mpMRI prior to ORP or RARP were retrospectively analyzed. Uni- and multivariable logistic regression models were used to identify anatomic characteristics for very early continence rates, defined as urine loss of $\leq 1 \mathrm{~g}$ in the PAD-test. Results Of 128 patients with mpMRI prior to surgery, 76 (59.4\%) underwent RARP vs. 52 (40.6\%) ORP. In total, median USL was 15,15 and $10 \mathrm{~mm}$ in the sagittal, coronal and axial dimensions. After stratification according to very early continence in the PAD-test $(\leq 1 \mathrm{~g}$ vs. $>1 \mathrm{~g})$, continent patients had significantly more frequently Lee type D (71.4 vs. 54.4\%) and C (14.3 vs. $7.6 \%, p=0.03)$. In multivariable logistic regression models, the sagittal median USL (odds ratio [OR] 1.03) and Lee type C (OR: 7.0) and D (OR: 4.9) were independent predictors for achieving very early continence in the PAD-test. Conclusion Patients' individual anatomical characteristics in mpMRI prior to radical prostatectomy can be used to predict very early continence. Lee type $\mathrm{C}$ and $\mathrm{D}$ suggest being the most favorable anatomical characteristics. Moreover, longer sagittal median USL in mpMRI seems to improve very early continence rates.
\end{abstract}

Keywords ORP $\cdot$ RARP $\cdot$ Lee type, functional outcome $\cdot$ PAD-test

Mike Wenzel and Felix Preisser contributed to the work equally.

Mike Wenzel

Mike.Wenzel@kgu.de

1 Department of Urology, University Hospital Frankfurt, Goethe University, Frankfurt am Main, Germany

2 Cancer Prognostics and Health Outcomes Unit, Division of Urology, University of Montreal Health Center, Montreal, QC, Canada

3 Department of Diagnostic and Interventional Radiology, University Hospital Frankfurt, Frankfurt am Main, Germany

4 Martini-Klinik Prostate Cancer Center, University Hospital Hamburg-Eppendorf, Hamburg, Germany

5 Department of Urology, University Hospital Hamburg-Eppendorf, Hamburg, Germany

\section{Introduction}

In the treatment of prostate cancer with radical prostatectomy for local tumor control, sufficient postoperative functional outcomes - mainly continence and potency — are of major importance to provide high quality of life in men [1-4]. In the recent years, several surgical techniques, such as nerve-sparing procedures or preservation of the "FullFunctional-Length-Urethra" (FFLU) have been investigated with the aim of improving early postoperative continence rates after open (ORP) and robotic-assisted radical prostatectomy (RARP) [5-9].

In shared decision making with prostate cancer patients regarding treatment choice of radical prostatectomy vs. radiation therapy, it is crucial to provide information and predict the postoperative probability of functional outcomes 
like early continence. Several risk factors for higher risk of postoperative incontinence as for example non-organ confined disease, age, non-nerve-sparing surgery, prostate volume or Gleason score have been previously described [10, 11]. Moreover, multiparametric magnetic resonance imaging (mpMRI) is nowadays frequently employed for prostate cancer diagnostics, staging and surgical planning purposes and also individual anatomical characteristics can be visualized and analyzed according to their influence on continence rates after radical prostatectomy [12]. For example, the urethral sphincter length measured in the preoperative mpMRI and also the variation of the prostatic apex shape, classified as four types as previously described by Lee et al. may yield the potential to predict continence rates [13-17]. Nonetheless, further validation of those predictors and interindividual anatomical characteristics is needed, since mpMRI quality improved in the recent years and RARP approach may allow better visibility of anatomical structures. Moreover, to the best of our knowledge the influence of these characteristics on very early continence, defined as urine loss in the validated PAD-test within $24 \mathrm{~h}$ after catheter removal, has never been investigated.

We addressed this void in analyzing our institutional radical prostatectomy database according to very early continence rates of the most recent patients who underwent mpMRI prior to ORP or RARP between 01/2018 and $12 / 2019$. We hypothesized that very early postoperative continence rates differ according to the apex shape of the prostate, classified as Lee types A-D, as well as urethral sphincter length after ORP and RARP.

\section{Methods}

\section{Study population}

After approval of the local ethic committee, we identified all patients $(n=334)$ who underwent radical prostatectomy for biopsy confirmed prostate cancer at the University Hospital Frankfurt between 01/2018 and 12/2019 in the institutional radical prostatectomy database. Patients without preoperative mpMRI were excluded $(n=206)$. All surgeons in this patient cohort were experienced surgeons trained in highvolume prostate cancer centers. Radical prostatectomy was performed as ORP or RARP using FFLU-technique and frozen sections for nerve-sparing surgery, as previously described $[6,18]$.

\section{mpMRI prior to radical prostatectomy: Lee type definition and urethral sphincter length}

mpMRI acquisition was performed as recommended (European Society of Urogenital Radiology ESUR guidelines) and previously described [19]. Moreover, according to the previous publication by Lee et al. and its value for continence prediction, prostatic apex shape in the mpMRI was classified as Lee type A-D in T2-weighted sequences [17]: Lee type A was defined as a prostatic apex overlapping the membranous urethra anteriorly and posteriorly. Lee type B and C were, respectively, defined as an overlap of the prostatic apex of the anterior or posterior membranous urethra. In addition, Lee type $\mathrm{D}$ was defined as no observed overlap of the prostatic apex over the membranous urethra in the mpMRI.

Moreover, urethral sphincter length and diameter were measured (in millimeters [mm]) in sagittal, coronal and axial directions in preoperative mpMRI (Fig. 1). All mpMRI analyses were primarily performed by an experienced researcher with special training in uropathological imaging in a blinded fashion with regard to the study endpoint, supervised by a board-certificated radiologist.

\section{Continence rates after radical prostatectomy}

Very early continence after ORP and RARP was defined as continence within $24 \mathrm{~h}$ after removal of the transurethral catheter. The catheter was removed after unsuspicious cystogram, routinely performed between the fifth to the seventh day after surgery. A standardized assessment of very early continence rates was performed in all patients with the PAD-test within $24 \mathrm{~h}$ after catheter removal, as previously described [10]. The PAD-test describes a comprehensible

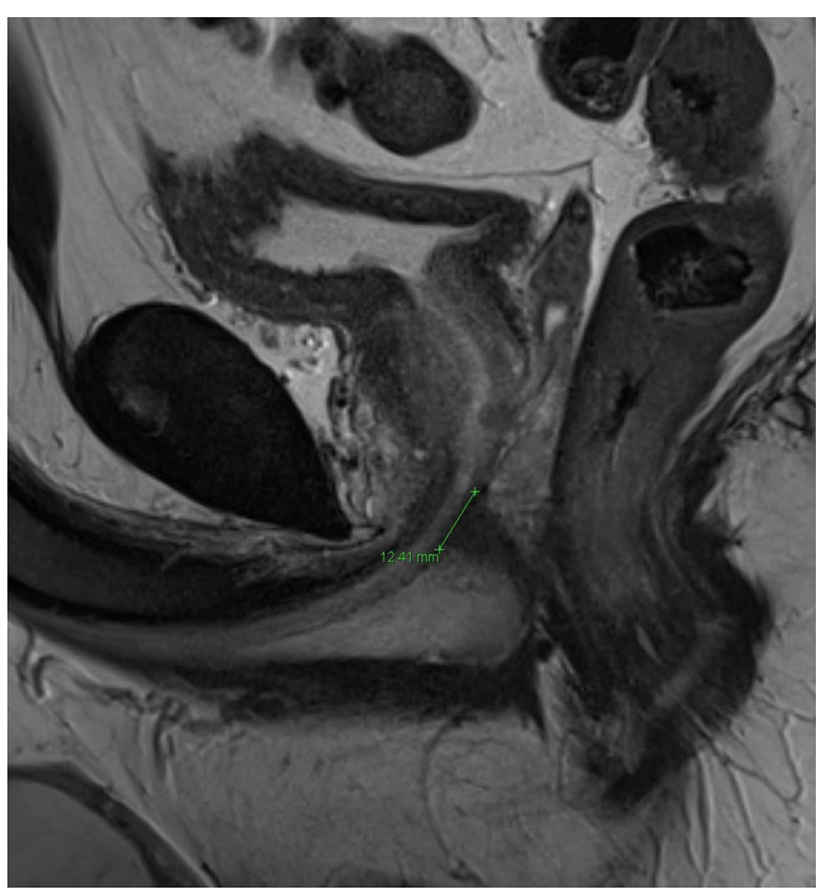

Fig. 1 Urethral sphincter measurement in sagittal dimension in a multiparametric MRI prior to radical prostatectomy 
and validated test which measures the amount of involuntary urine loss while performing predefined physical activities in $1 \mathrm{~h}$ (e.g., coughing, walking, climbing stairs). Prior to the PAD-test, a pre-weighed collecting device was given to the patient and after physically activities, the collecting device was removed and reweighed. Very early continence was defined as a urine loss $\leq 1 \mathrm{~g}$ in the PAD-test [20].

\section{Statistical analyses}

Descriptive statistics included frequencies and proportions for categorical variables. Means, medians and interquartile ranges (IQR) were reported for continuously coded variables. The Chi-square test was used for statistical significance in proportions' differences. The $t$ test and Kruskal-Wallis test examined the statistical significance of means' and distributions' differences.

Univariable and multivariable logistic regression models were fitted to test the relationship between Lee types A-D and urethral sphincter length in the preoperative mpMRI on the very early postoperative continence. Logistic regression models were set for the covariables age, prostate volume, pT-stage, nerve-sparing procedure and surgical approach (ORP vs. RARP). Statistically significant variables in univariable models were used for additional multivariable logistic regression models. All tests were two sided with a level of significance set at $p<0.05$ and $\mathrm{R}$ software environment for statistical computing and graphics (version 3.4.3) was used for all analyses.

\section{Results}

\section{Descriptive baseline characteristics}

Between 01/2018 and 12/2019, 334 patients underwent radical prostatectomy for biopsy confirmed prostate cancer at the University Hospital Frankfurt. Of these, 128 patients had a preoperative mpMRI prior to radical prostatectomy and were included in the final analyses. In total, 76 (59.4\%) patients underwent RARP vs. 52 (40.6\%) ORP. Median age and median PSA were 66 (IQR: $60-70)$ and $8.0 \mathrm{ng} / \mathrm{ml}$ (IQR 6.0-11.5), respectively. In our cohort, 38 (29.7\%), 16 (12.2\%) and 29 (22.7\%) patients inherited unfavorable characteristics such as high risk D'amico score, cT-stage $\geq 2 \mathrm{c}$ and Gleason Score 8-10 (ISUP 4-5), respectively. Neurovascular bundle preservation was performed on both sides in 92 (71.9\%) and at one side in 24 (18.8\%) patients. Overall, 49 (38.3\%) patients had a very early continence with $\leq 1 \mathrm{~g}$ urine loss in the PAD-test. Further stratification was performed according to PAD-test $\leq 1 \mathrm{~g}$ vs. $>1 \mathrm{~g}$ (Table 1 ).

\section{mpMRI characteristics: urethral length and Lee types}

Median length of the urethral sphincter was $15 \mathrm{~mm}$ (IQR 12-17), $15 \mathrm{~mm}$ (IQR 12-17) in the entire cohort in the sagittal and coronal dimension, respectively. Moreover, median axial and coronal diameter of the urethral sphincter were $9 \mathrm{~mm}$ (IQR 8-10) and $10 \mathrm{~mm}$ (IQR 9-11). Proportions of Lee type A, B, C and D were 21 (16.4\%), 16 (12.5\%), 13 $(10.2 \%)$ and $78(60.9 \%)$ in the entire cohort.

After stratification according to very early continence in the PAD-test ( $\leq 1 \mathrm{~g}$ vs. $>1 \mathrm{~g}$ ), a significant longer median length of the urethral sphincter in sagittal dimension was observed in the group with a PAD-test $\leq 1 \mathrm{~g}$ vs. $>1 \mathrm{~g}(15 \mathrm{~mm}$ [IQR $14-18$ ] vs. 15.0 [IQR $12-17, p=0.03$ ]. Conversely, no differences in the coronal and axial dimensions of the urethral length were observed between both groups (all $p>0.05$ ). Furthermore, patients with a PAD-test $\leq 1 \mathrm{~g}$ after catheter removal had significantly more frequently Lee type D (71.4 vs. $54.4 \%)$ and C (14.3 vs. $7.6 \%, p=0.03)$ than patients with a PAD-test $>1 \mathrm{~g}$. Conversely, patients with a PAD-test $>1 \mathrm{~g}$ had significantly more frequently Lee type A (22.8 vs. $6.1 \%$ ) and $\mathrm{B}(15.2$. vs. $8.2 \%)$ in the mpMRI prior to radical prostatectomy than patients with a PAD-test $\leq 1 \mathrm{~g}$ $(p=0.03)$.

\section{PAD-test $\leq 1 \mathrm{~g}$ vs. PAD-test $>1 \mathrm{~g}$}

In univariable logistic regression analyses (Table 2), the length of the urethral sphincter in sagittal dimension (odds ratio [OR] 1.2), prostatic apex Lee type C (OR 7.0) and D (OR 4.9) and RARP (OR 2.7) were significant predictors for a very early continence in the PAD-test (all $p<0.05$ ). In multivariable analyses, median length of the urethral sphincter in the sagittal dimension (OR 1.03), as well as Lee type $\mathrm{C}$ (OR 1.5) and D (OR 1.3) were independent predictors for very early continence, defined as PAD-test $\leq 1 \mathrm{~g}$ (all $p<0.05$ ). Conversely, RARP or ORP had no influence on very early continence rates in multivariable logistic regression models $(p>0.05)$.

\section{Discussion}

We hypothesized that very early postoperative continence rates differ according to the apex shape of the prostate, classified as Lee types A-D, as well as the urethral sphincter length in mpMRI prior to surgery. We tested this hypothesis in our institutional radical prostatectomy database and made several noteworthy observations.

First, we made important observations according to the prostatic apex shape and the median urethral sphincter length. Specifically, the most common prostatic apex shape 
Table 1 Patient characteristics of 128 patients who underwent radical prostatectomy for biopsy confirmed prostate cancer between 01/2018 and 12/2019 at the University Hospital Frankfurt and received preop- erative mpMRI, stratified by very early continence rates, classified by PAD-test (0-1 g vs. $>1 \mathrm{~g})$

\begin{tabular}{|c|c|c|c|c|}
\hline Variable & $\begin{array}{l}\text { Overall } \\
n=128\end{array}$ & $\begin{array}{l}\text { PAD-test } 0-1 \mathrm{~g} \\
n=49(38.3 \%)\end{array}$ & $\begin{array}{l}\text { PAD-test }>1 \mathrm{~g} \\
n=79(61.7 \%)\end{array}$ & $p$ value \\
\hline \multicolumn{5}{|l|}{ Age, in years } \\
\hline Median (IQR) & $66(60-70)$ & $65(60-68)$ & $66(60-72)$ & 0.3 \\
\hline \multicolumn{5}{|l|}{ PSA, in ng/ml } \\
\hline Median (IQR) & $8.0(6.0-11.5)$ & $7.0(6.0-10.0)$ & $8.0(6.0-14.0)$ & 0.04 \\
\hline \multicolumn{5}{|c|}{ Length of urethral sphincter, sagittal, in mm } \\
\hline Median (IQR) & $15(12-17)$ & $15(14-18)$ & $15(12-17)$ & 0.03 \\
\hline \multicolumn{5}{|c|}{ Length of urethral sphincter, coronal, in mm } \\
\hline Median (IQR) & $15(12-17)$ & $15(13-18)$ & $15(12-17)$ & 0.1 \\
\hline \multicolumn{5}{|c|}{ Diameter of urethral sphincter, axial, in mm } \\
\hline Median (IQR) & $10(9-11)$ & $10(9-11)$ & $10(9-11)$ & 0.9 \\
\hline \multicolumn{5}{|c|}{ Diameter of urethral sphincter, coronal, in mm } \\
\hline Median (IQR) & $9(8-10)$ & $9(8-10)$ & $9(8-10)$ & 0.2 \\
\hline \multicolumn{5}{|l|}{ Prostate volume, in ml } \\
\hline Median (IQR) & $40(30-50)$ & $40(30-45)$ & $40(29-50)$ & 0.8 \\
\hline \multicolumn{5}{|l|}{ D’Amico score, $n(\%)$} \\
\hline Low & $12(9.4)$ & $9(18.4)$ & $3(3.8)$ & $<0.01$ \\
\hline Intermediate & $76(59.4)$ & $31(63.3)$ & $45(57.0)$ & \\
\hline High & $38(29.7)$ & $8(16.3)$ & $30(38.0)$ & \\
\hline Missing information & $2(1.6)$ & $1(2.0)$ & $1(1.3)$ & \\
\hline \multicolumn{5}{|l|}{ Lee type, $n(\%)$} \\
\hline A & $21(16.4)$ & $3(6.1)$ & $18(22.8)$ & 0.03 \\
\hline B & $16(12.5)$ & $4(8.2)$ & $12(15.2)$ & \\
\hline $\mathrm{C}$ & $13(10.2)$ & $7(14.3)$ & $6(7.6)$ & \\
\hline $\mathrm{D}$ & $78(60.9)$ & $35(71.4)$ & $43(54.4)$ & \\
\hline \multicolumn{5}{|l|}{ pT-stage, $n(\%)$} \\
\hline $\mathrm{pT} 2$ & $68(53.1)$ & $30(61.2)$ & $38(48.1)$ & 0.2 \\
\hline$\geq \mathrm{pT} 3$ & $60(46.9)$ & $19(38.8)$ & $41(51.9)$ & \\
\hline \multicolumn{5}{|c|}{ Surgical approach, $n(\%)$} \\
\hline ORP & $52(40.6)$ & $13(26.5)$ & $39(49.4)$ & 0.02 \\
\hline RARP & $76(59.4)$ & $36(73.5)$ & $40(50.6)$ & \\
\hline \multicolumn{5}{|l|}{ Nerve sparing, $n(\%)$} \\
\hline None & $12(9.4)$ & $1(2.0)$ & $11(13.9)$ & 0.1 \\
\hline Unilateral & $24(18.8)$ & $8(16.3)$ & $16(20.3)$ & \\
\hline Bilateral & $92(71.9)$ & $40(81.6)$ & $52(65.8)$ & \\
\hline \multicolumn{5}{|l|}{ Surgical margin, $n(\%)$} \\
\hline Negative & $88(68.8)$ & $37(75.5)$ & $51(64.6)$ & 0.3 \\
\hline Positive & $40(31.2)$ & $12(24.5)$ & $28(35.4)$ & \\
\hline
\end{tabular}

$I Q R$ interquartile range, $P S A$ initial prostate-specific antigen

was Lee type D (61\%), followed by A (16\%), B (13\%) and C (13\%). Moreover, longest median urethral sphincter length was measured in sagittal dimension and was $15.1 \mathrm{~mm}$ (IQR 12.4-17.1). These observations are quite interesting, since geographical differences may exist regarding to the prostatic apex shape and the length of the median urethral sphincter. For example, in the first described classification of the prostatic apex shape overlapping the urethra by Lee et al., the majority of patients harbored Lee type A (38\%) and the minority Lee type C (15\%). Since Lee et al. demonstrated that the different anatomical shapes of the prostate apex influence urinary continence after radical prostatectomy, it is very important to investigate those geographical differences for comparisons and real-world application [17]. Moreover, also the median urethral length in sagittal dimension seems to differ across geographical areas. For example, in several 
Table 2 Univariable and multivariable logistic regression models predicting very early continence after radical prostatectomy, defined as PADtest $0-1 \mathrm{~g}$

\begin{tabular}{|c|c|c|c|c|c|c|c|c|}
\hline & \multicolumn{4}{|c|}{ Univariable analysis } & \multicolumn{4}{|c|}{ Multivariable analysis } \\
\hline & OR & CI $2.5 \%$ & CI $97.5 \%$ & $p$ value & OR & $2.5 \%$ & $97.5 \%$ & $p$ value \\
\hline Length of urethral sphincter, sagittal, in mm & 1.15 & 1.03 & 1.29 & 0.01 & 1.03 & 1.01 & 1.05 & 0.037 \\
\hline Length of urethral sphincter, coronal, in mm & 1.12 & 1.00 & 1.27 & 0.06 & - & - & - & - \\
\hline Diameter of urethral sphincter, axial, in mm & 1.02 & 0.81 & 1.28 & 0.9 & - & - & - & - \\
\hline Diameter of urethral sphincter, coronal, in $\mathrm{mm}$ & 1.18 & 0.94 & 1.49 & 0.2 & - & - & - & - \\
\hline Lee type A & $\operatorname{Ref}(1.0)$ & - & - & - & - & - & - & - \\
\hline B & 2.00 & 0.38 & 11.74 & 0.4 & 1.15 & 0.85 & 1.55 & 0.4 \\
\hline $\mathrm{C}$ & 7.00 & 1.47 & 41.67 & 0.02 & 1.53 & 1.10 & 2.11 & 0.01 \\
\hline $\mathrm{D}$ & 4.88 & 1.50 & 22.06 & 0.02 & 1.27 & 1.01 & 1.59 & 0.04 \\
\hline Age & 0.98 & 0.93 & 1.03 & 0.4 & - & - & - & - \\
\hline Prostate volume, in $\mathrm{ml}$ & 0.99 & 0.96 & 1.01 & 0.3 & - & - & - & - \\
\hline Surgical approach ORP & Ref (1.0) & - & - & - & - & - & - & - \\
\hline RARP & 2.70 & 1.27 & 6.00 & 0.01 & 1.17 & 0.99 & 1.39 & 0.1 \\
\hline pT2 stage & $\operatorname{Ref}(1.0)$ & - & - & - & - & - & - & - \\
\hline pT3-4 & 0.59 & 0.28 & 1.20 & 0.1 & - & - & - & - \\
\hline No nerve sparing & Ref (1.0) & - & - & - & - & - & - & - \\
\hline Uni-/bilateral nerve sparing & 7.76 & 1.44 & 144.3 & 0.1 & - & - & - & - \\
\hline
\end{tabular}

$O R$ Odds ratio, $C I$ confidence interval, $P S A$ prostate-specific antigen, $O R P$ open radical prostatectomy, $R A R P$ robotic-assisted radical prostatectomy

studies and a meta-analysis by Mungovan et al., the median urethral length was mostly comparable to our findings in North American and European studies, while in Asian men, the median urethral sphincter length seemed to be shorter and ranged from 10.4 to $13.3 \mathrm{~mm}$ [14, 21, 22]. However, when determining Lee type and measuring urethral sphincter length, interobserver variability might be present and influence the results.

Second, we also made important observations after stratifying our cohort by PAD-test result $\leq 1 \mathrm{~g}$ vs. $>1 \mathrm{~g}$. Specifically, patients with very early continence after catheter removal after radical prostatectomy harbored significantly more often none (Lee type D, 71 vs. $54 \%$ ) or a posterior overlap (Lee type C, 14 vs. $8 \%$ ) of the prostatic apex on the membranous urethra. Moreover, Lee type $\mathrm{C}$ and $\mathrm{D}$ were independent positive predictors for very early continence in multivariable logistic regression models. Comparing our findings with other studies, highest rates of urinary continence in the first publication by Lee et al. [17] were found in Lee type B or C 3 months after surgery. Since surgical techniques have impressively improved since the time of publication by Lee et al. and geographical differences in patients according to Lee types may exist as mentioned above, as well as the different analyzed timepoints for continence, the heterogeneity of both studies does not allow direct comparisons. It can be assumed that Lee type D (no prostatic apex overlap on the urethra) might be most favorable anatomical variant for surgeons to perform surgical techniques such as
FFLU and this might reflect the high rates of very early postoperative continence [10, 22].

In addition, a significant longer median urethral length in the sagittal dimension in the mpMRI prior to radical prostatectomy was observed in patients with very early continence rates (PAD-test $\leq 1 \mathrm{~g}$ ). Moreover, median sagittal urethral length was also an independent predictor for very early continence (OR 1.03) in multivariable logistic regression models after adjusting for the influence of surgical approach and techniques. These findings are very important since it can help clinicians to predict very early continence rates after catheter removal with the preoperative MRI and can provide a realistic preoperative continence prediction for the patients, despite the influence of for example, surgical approach, FFLU or nervesparing technique [5]. To the best of our knowledge, no previous study investigated the effect of Lee types and urethral sphincter length on very early continence outcomes, defined as continence measurement within $24 \mathrm{~h}$ after removal of the catheter after radical prostatectomy. In consequence, our data cannot be directly compared to other investigations. However, several studies reported a significant influence of the urethral length on continence rates, using different follow-up durations and continence definitions. For example, Kim et al. reported a significant influence of median urethral length on continence rates, defined as a PAD-test $<2 \mathrm{~g}, 3$ months after surgery [21]. Moreover, Ko et al. reported a significant influence of a 
median urethral length $>14 \mathrm{~mm}$ on 30 -day continence rates, defined as pad-free continence [23]. It is of note that since we used a stricter cutoff for the definition of continence ( $\leq 1 \mathrm{~g}$ in PAD-test) and the PAD-test was performed within $24 \mathrm{~h}$ after catheter removal, we believe that our findings even stronger emphasize and corroborate the effect of the urethral length on the urinary continence in an unbiased fashion.

Finally, we also made important observations between the comparison of ORP vs. RARP on continence rates. Patients with a PAD-test $\leq 1 \mathrm{~g}$ underwent more frequently RARP than ORP. However, after multivariable adjustment for differences in patient characteristics, the surgical approach did not influence very early continence rates, defined as PADtest result of $\leq 1 \mathrm{~g}$ within $24 \mathrm{~h}$ after catheter removal. These findings are also important in the context of an often-stated argument of better visibility of anatomical structures with a RARP approach [24]. However, the same findings of no difference between RARP and ORP approach, after adjustment for age differences, were observed in a recently published comparison of a Martini-Klinik series with 10,790 radical prostatectomy patients regarding to continence rates 3 and 12 months after surgery [25].

Our study has several limitations and should first be interpreted in its retrospective design. Second, sample size limitations might impair statistical significance in some of the analyses. Third, even though all mpMRI were analyzed by an experienced specialist in urologic imaging who was supervised by a board-certificated radiologist interobserver variability cannot be ruled out. Moreover, only patients with an mpMRI and catheter removement during the in-hospital stay were included, which may have led to a selection bias. Fourth, both surgical approaches (ORP and RARP) represent methods with different learning curves and surgery was performed by different surgeons. Those factors may have influenced very early continence rates. Unfortunately, other endpoints with longer follow-up duration were not available. Prospective studies are needed to further validate or reject our findings.

Taken together, our findings demonstrate that the anatomical prostate apex shape of Lee type $\mathrm{C}$ and $\mathrm{D}$ in the mpMRI prior to surgery provided the best prediction for very early continence rates after catheter removal after radical prostatectomy. Moreover, our data also demonstrated that the median urethral length of the sphincter in sagittal dimension in mpMRI predicted very early continence rates. According to the comparison of ORP vs. RARP, no differences existed according to very early continence rates after consideration of Lee types and urethral length. These findings are important with regard to the increasing numbers of performed mpMRI prior to radical prostatectomy and should be considered by clinicians for patients' information and surgical planning purposes.
Author contributions WM: manuscript writing - original draft preparation, protocol/project development, and data analysis. PF: manuscript writing — original draft preparation, protocol/project development, and data analysis. MM: manuscript writing/editing. TLH: data collection or management. WMN: data collection or management. HB: data collection or management. HC: manuscript writing/editing. BS: data analysis. BB: data analysis. WC: formal analysis and investigation. TD: supervision. HH: supervision. GM: supervision. RF: writing-writing/editing. BA: writing — writing/editing. KPI: supervision and writing —writing/ editing. CFKH: protocol/project development. KLA: protocol/project development and writing — writing/editing. MM: data analysis, manuscript writing — original draft preparation, and supervision.

Funding Open Access funding enabled and organized by Projekt DEAL. The authors declare that this study had no funding.

Code availability On request. Used software R statistics (version 3.6.1).

\section{Compliance with ethical standards}

Conflict of interest The authors declare that they have no conflict of interest.

Ethics approval This research study was conducted retrospectively from data obtained for clinical purposes. We consulted extensively with the local Ethics Committee of University Hospital Frankfurt and ethical approval was given (approval number: SUG-1-2020).

Consent to participate Not applicable.

Consent for publication Not applicable.

Availability of data and material All datasets generated for this study are included in the manuscript.

Open Access This article is licensed under a Creative Commons Attribution 4.0 International License, which permits use, sharing, adaptation, distribution and reproduction in any medium or format, as long as you give appropriate credit to the original author(s) and the source, provide a link to the Creative Commons licence, and indicate if changes were made. The images or other third party material in this article are included in the article's Creative Commons licence, unless indicated otherwise in a credit line to the material. If material is not included in the article's Creative Commons licence and your intended use is not permitted by statutory regulation or exceeds the permitted use, you will need to obtain permission directly from the copyright holder. To view a copy of this licence, visit http://creativecommons.org/licenses/by/4.0/.

\section{References}

1. Mottet N, Cornford P, Van den Bergh RCN et al. (2020) EAU Guidelines Edn. presented at the EAU Annual Congress Amsterdam 2020. ISBN 978-94-92671-07-3

2. Mohler JL, Antonarakis ES, Armstrong AJ et al (2019) Prostate cancer, version 2.2019, NCCN clinical practice guidelines in oncology. J Natl Compr Cancer Netw JNCCN 17(5):479-505. https://doi.org/10.6004/jncen.2019.0023

3. Donovan JL, Hamdy FC, Lane JA et al (2016) Patient-reported outcomes after monitoring, surgery, or radiotherapy for prostate cancer. N Engl J Med 375(15):1425-1437. https://doi.org/10. 1056/NEJMoa1606221 
4. Nicolaisen M, Müller S, Patel HRH, Hanssen TA (2014) Quality of life and satisfaction with information after radical prostatectomy, radical external beam radiotherapy and postoperative radiotherapy: a long-term follow-up study. J Clin Nurs 23(2324):3403-3414. https://doi.org/10.1111/jocn.12586

5. Schlomm T, Tennstedt P, Huxhold C et al (2012) Neurovascular structure-adjacent frozen-section examination (NeuroSAFE) increases nerve-sparing frequency and reduces positive surgical margins in open and robot-assisted laparoscopic radical prostatectomy: experience after 11,069 consecutive patients. Eur Urol 62(2):333-340. https://doi.org/10.1016/j.eururo.2012.04.057

6. Preisser F, Theissen L, Wild P et al (2019) Implementation of intraoperative frozen section during radical prostatectomy: shortterm results from a German tertiary-care center. Eur Urol Focus. https://doi.org/10.1016/j.euf.2019.03.007

7. Lee J, Kim HY, Goh HJ et al (2020) Retzius sparing robot-assisted radical prostatectomy conveys early regain of continence over conventional robot-assisted radical prostatectomy: a propensity score matched analysis of 1863 patients. J Urol 203(1):137-144. https:// doi.org/10.1097/JU.0000000000000461

8. Vis AN, van der Poel HG, Ruiter AEC et al (2019) Posterior, anterior, and periurethral surgical reconstruction of urinary continence mechanisms in robot-assisted radical prostatectomy: a description and video compilation of commonly performed surgical techniques. Eur Urol 76(6):814-822. https://doi.org/10.1016/j.eururo. 2018.11.035

9. Egan J, Marhamati S, Carvalho FLF et al (2020) Retzius-sparing robot-assisted radical prostatectomy leads to durable improvement in urinary function and quality of life versus standard robotassisted radical prostatectomy without compromise on oncologic efficacy: single-surgeon series and step-by-step guide. Eur Urol. https://doi.org/10.1016/j.eururo.2020.05.010

10. Theissen L, Preisser F, Wenzel M et al (2019) Very early continence after radical prostatectomy and its influencing factors. Front Surg 6:60. https://doi.org/10.3389/fsurg.2019.00060

11. Hatiboglu G, Teber D, Tichy D et al (2016) Predictive factors for immediate continence after radical prostatectomy. World J Urol 34(1):113-120. https://doi.org/10.1007/s00345-015-1594-4

12. Marenco J, Orczyk C, Collins T, Moore C, Emberton M (2019) Role of MRI in planning radical prostatectomy: what is the added value? World J Urol 37(7):1289-1292. https://doi.org/10.1007/ s00345-019-02762-2

13. Kitamura K, China T, Kanayama M et al (2019) Significant association between urethral length measured by magnetic resonance imaging and urinary continence recovery after robot-assisted radical prostatectomy. Prostate Int 7(2):54-59. https://doi.org/ 10.1016/j.prnil.2018.06.003

14. Mungovan SF, Sandhu JS, Akin O, Smart NA, Graham PL, Patel MI (2017) Preoperative membranous urethral length measurement and continence recovery following radical prostatectomy: a systematic review and meta-analysis. Eur Urol 71(3):368-378. https://doi.org/10.1016/j.eururo.2016.06.023
15. Kim M, Park M, Pak S et al (2019) Integrity of the urethral sphincter complex, nerve-sparing, and long-term continence status after robotic-assisted radical prostatectomy. Eur Urol Focus 5(5):823830. https://doi.org/10.1016/j.euf.2018.04.021

16. Hakimi AA, Faleck DM, Agalliu I, Rozenblit AM, Chernyak V, Ghavamian R (2011) Preoperative and intraoperative measurements of urethral length as predictors of continence after robotassisted radical prostatectomy. J Endourol 25(6):1025-1030. https://doi.org/10.1089/end.2010.0692

17. Lee SE, Byun S-S, Lee HJ et al (2006) Impact of variations in prostatic apex shape on early recovery of urinary continence after radical retropubic prostatectomy. Urology 68(1):137-141. https:// doi.org/10.1016/j.urology.2006.01.021

18. Wenzel M, Preisser F, Theissen LH et al (2020) The effect of adverse patient characteristics on perioperative outcomes in open and robot-assisted radical prostatectomy. Front Surg 7:584897. https://doi.org/10.3389/fsurg.2020.584897

19. Barentsz JO, Weinreb JC, Verma S et al (2016) Synopsis of the PI-RADS v2 guidelines for multiparametric prostate magnetic resonance imaging and recommendations for use. Eur Urol 69(1):41-49. https://doi.org/10.1016/j.eururo.2015.08.038

20. Hahn I, Fall I (1991) Objective quantification of stress urinary incontinence: a short, reproducible, provocative pad-test. Neurourol Urodyn 10:475-481. https://doi.org/10.1002/nau.1930100503

21. Kim LHC, Patel A, Kinsella N, Sharabiani MTA, Ap Dafydd D, Cahill D (2020) Association between preoperative magnetic resonance imaging-based urethral parameters and continence recovery following robot-assisted radical prostatectomy. Eur Urol Focus 6(5):1013-1020. https://doi.org/10.1016/j.euf.2019.01.011

22. Tienza A, Hevia M, Benito A, Pascual JI, Zudaire JJ, Robles JE (2015) MRI factors to predict urinary incontinence after retropubic/laparoscopic radical prostatectomy. Int Urol Nephrol 47(8):1343-1349. https://doi.org/10.1007/s11255-015-1019-8

23. Ko YH, Huynh LM, See K, Lall C, Skarecky D, Ahlering TE (2020) Impact of surgically maximized versus native membranous urethral length on 30-day and long-term pad-free continence after robot-assisted radical prostatectomy. Prostate Int 8(2):55-61. https://doi.org/10.1016/j.prnil.2019.12.005

24. Beyer B, Kühne K, Böhm K et al (2015) DaVinci robot-assisted laparoscopic prostatectomy: benefit for obese men?-a matchedpair analysis. Urol Ausg A 54(1):34-40. https://doi.org/10.1007/ s00120-014-3589-y

25. Haese A, Knipper S, Isbarn H et al (2019) A comparative study of robot-assisted and open radical prostatectomy in 10790 men treated by highly trained surgeons for both procedures. BJU Int 123(6):1031-1040. https://doi.org/10.1111/bju.14760

Publisher's Note Springer Nature remains neutral with regard to jurisdictional claims in published maps and institutional affiliations. 